\title{
Letter to the editors regarding comments by Ghirardello et al. on the rasburicase article
}

\author{
David Hobbs • Gina-Marie Barletta • Jin Chung • \\ Julia Steinke • Timothy Bunchman
}

Received: 3 March 2010 / Accepted: 8 March 2010/Published online: 9 April 2010

(C) IPNA 2010

Sirs,

We appreciate the comments by Ghirardello et al. [1] concerning their experience with rasburicase in neonates with hyperuricemia and acute kidney injury (AKI). They reference their unpublished data and assert that the concomitant improvements in urinary output and serum creatinine observed in our study with the lowering of uric acid were likely the result of volume reconstitution, not a therapeutic effect of rasburicase [2].

Several tumor lysis syndrome studies demonstrating the utility of rasburicase in the absence of volume reconstitution have demonstrated significant improvement of serum creatinine with uric acid [3-5]. We cannot definitively state that rasburicase improved the renal function in our hyperuricemic infants with AKI. When these findings are taken together, the report by Ghirardello et al. contrasting published studies demonstrating a potential renal benefit of treatment with rasburicase identifies the need for a prospective study targeting uric acid reduction to prove or disprove its benefit to the infant with AKI.

\section{References}

1. Ghirardello S, Ardissino G, Mastrangelo A, Mosca F (2010) Rasburicase in the treatment of hyperuricemia of newborns. Pediatr Nephrol. doi:10.1007/s00467-010-1515-0

2. Hobbs DJ, Steinke JM, Chung JY, Barletta GM, Bunchman TE (2010) Rasburicase improves hyperuricemia in infants with acute kidney injury. Pediatr Nephrol 25:305-309

3. Goldman SC, Holcenberg JS, Finklestein JZ, Hutchinson R, Kreissman S, Johnson FL, Tou C, Harvey E, Morris E, Cairo MS (2001) A randomized comparison between rasburicase and allopurinol in children with lymphoma or leukemia at high risk for tumor lysis. Blood 97:2998-3003

4. Pui CH, Mahmoud HH, Wiley JM, Woods GM, Leverger G, Camitta B, Hastings C, Blaney SM, Relling MV, Reaman GH (2001) Recombinant urate oxidase for the prophylaxis or treatment of hyperuricemia in patients with leukemia or lymphoma. J Clin Oncol 19:697-704

5. Coiffier B, Mounier N, Bologna S, Fermé C, Tilly H, Sonet A, Christian B, Casasnovas O, Jourdan E, Belhadj K, Herbrecht R, Groupe d'Etude des Lymphomes de l'Adulte Trial on Rasburicase Activity in Adult Lymphoma (2003) Efficacy and safety of rasburicase (recombinant urate oxidase) for the prevention and treatment of hyperuricemia during induction chemotherapy of aggressive nonHodgkin's lymphoma: results of the GRAAL1 (Groupe d'Etude des Lymphomes de l'Adulte Trial on Rasburicase Activity in Adult Lymphoma) study. J Clin Oncol 21:4402-4406

D. Hobbs $\cdot$ G.-M. Barletta $\cdot$ J. Chung $\cdot$ J. Steinke $\cdot$

T. Bunchman $(\bowtie)$

Helen DeVos Children's Hospital,

221 Michigan Street NE, Suite 406,

Grand Rapids, MI 49503, USA

e-mail: Timothy.Bunchman@spectrum-health.org 